\title{
Proton decay of ${ }^{26} \mathrm{Si}$ via the ${ }^{28} \mathrm{Si}(\mathbf{p}, \mathrm{t})^{26} \mathrm{Si}$ Reaction and Implications for ${ }^{25} \mathrm{Al}(\mathbf{p}, \gamma)^{26} \mathrm{Si}$
}

\author{
K.A. Chipps* \\ Department of Physics and Astronomy, Rutgers University, Piscataway, NJ 08854, USA \\ and \\ Department of Physics, University of York, Heslington, York, UK \\ E-mail: kc612@york.ac.uk
}

D.W. Bardayan, J.F. Liang, C.D. Nesaraja, S.D. Pain, M.S. Smith

Physics Division, Oak Ridge National Laboratory, Oak Ridge, TN 37831, USA

K.Y. Chae, B.H. Moazen, S.T. Pittman, K.T. Schmitt

Department of Physics and Astronomy, University of Tennessee, Knoxville, TN 37996, USA

\section{J.A. Cizewski, P.D. O'Malley, W.A. Peters}

Department of Physics and Astronomy, Rutgers University, Piscataway, NJ 08854, USA

\section{R.L. Kozub}

Department of Physics, Tennessee Technological University, Cookeville, TN 38505, USA

\section{Matei}

Oak Ridge Associated Universities, Oak Ridge, TN 37830, USA

The rate of the ${ }^{25} \mathrm{Al}(\mathrm{p}, \gamma){ }^{26} \mathrm{Si}$ reaction in novae is important to the production of galactic ${ }^{26} \mathrm{Al}$, an important astronomical observable for its $1.809 \mathrm{MeV}$ gamma ray. ${ }^{26} \mathrm{Al}$ has been catalogued near massive stars and found in presolar grains. The ${ }^{25} \mathrm{Al}$ proton capture reaction serves to bypass ${ }^{26} \mathrm{Al}^{\text {g.s. }}$ production since the subsequent ${ }^{26} \mathrm{Si}$ beta decay preferentially populates instead the isomeric state of ${ }^{26} \mathrm{Al}$. Several recent studies have aimed to locate and characterize the first $\ell=0$ resonances in ${ }^{26} \mathrm{Si}$ above the ${ }^{25} \mathrm{Al}+\mathrm{p}$ threshold, which would dominate the reaction rate at nova temperatures. We have studied several resonances in ${ }^{25} \mathrm{Al}(\mathrm{p}, \gamma){ }^{26} \mathrm{Si}$ via the ${ }^{28} \mathrm{Si}(\mathrm{p}, \mathrm{t})^{26} \mathrm{Si}$ * reaction at the Holifield Radioactive Ion Beam Facility at Oak Ridge National Laboratory. In addition to measuring the angular distributions of the tritons, a partial implementation of the Oak Ridge Rutgers University Barrel Array (ORRUBA) was used to measure the coincident protons emitted from the decay of states in ${ }^{26} \mathrm{Si}$ above the proton threshold. We observe that the first $\ell=0$ state above the proton threshold decays essentially $100 \%$ of the time via proton emission, resulting in a proton branching ratio consistent with one.

11th Symposium on Nuclei in the Cosmos

19-23 July 2010

Heidelberg, Germany.

\footnotetext{
* Speaker.
} 


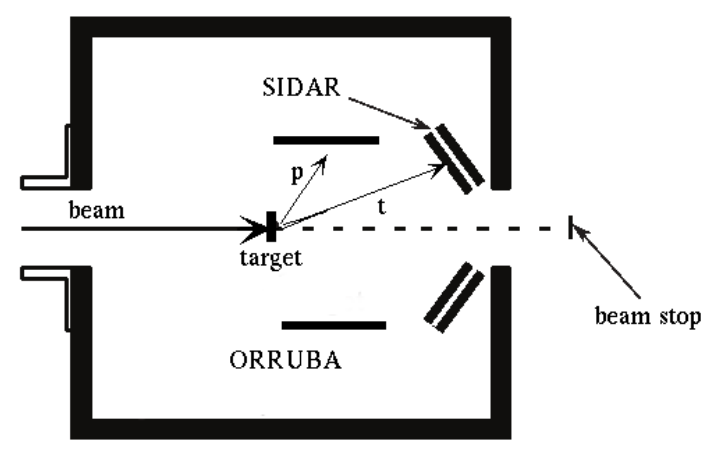

Figure 1: Experimental layout for the ${ }^{28} \mathrm{Si}(\mathrm{p}, \mathrm{t})^{26} \mathrm{Si}$ experiment, showing both ORRUBA and SIDAR (symmetric about the beam axis). The proton beam enters from the left; the proton labeled "p" in the diagram is the decay proton.

\section{Motivation}

The astrophysical rate of the ${ }^{25} \mathrm{Al}(\mathrm{p}, \gamma)^{26} \mathrm{Si}$ reaction is thought to greatly affect the production of galactic ${ }^{26} \mathrm{Al}$ in novae. Since the ${ }^{25} \mathrm{Al}(\mathrm{p}, \gamma)^{26} \mathrm{Si}\left(\beta^{+} v_{e}\right)^{26} \mathrm{Al}^{m}\left(\beta^{+} v_{e}\right)^{26} \mathrm{Mg}$ reaction sequence bypasses the production of ${ }^{26} \mathrm{Al}^{g s}$ [1] by populating the isomeric state, the rate of the ${ }^{25} \mathrm{Al}(\mathrm{p}, \gamma)^{26} \mathrm{Si}$ reaction (with respect to the beta-decay of ${ }^{25} \mathrm{Al}$ ) will play a role in how much ${ }^{26} \mathrm{Al}^{g s}$ is produced via the competing ${ }^{25} \mathrm{Al}\left(\beta^{+} v_{e}\right)^{25} \mathrm{Mg}(\mathrm{p}, \gamma)^{26} \mathrm{Al}^{g s}$ sequence. The ${ }^{25} \mathrm{Al}(\mathrm{p}, \gamma)^{26} \mathrm{Si}$ reaction rate is dominated at peak nova temperatures $(0.15-0.4 \mathrm{GK})$ by an $\ell=0$ resonance from the $5 / 2^{+25} \mathrm{Al}$ ground state (a $3^{+}$level in ${ }^{26} \mathrm{Si}$ ) with $E_{c . m .}<600 \mathrm{keV}$. Several recent measurements [1-8] have sought to find and characterize astrophysically relevant resonances in the ${ }^{25} \mathrm{Al}(\mathrm{p}, \gamma){ }^{26} \mathrm{Si}$ reaction, in order to precisely determine the effect on the production of galactic ${ }^{26} \mathrm{Al}$.

\section{Experiment}

A beam of protons at $40 \mathrm{MeV}$ was delivered by the Holifield Radioactive Ion Beam Facility (HRIBF) in order to study the resonances of significant astrophysical interest. The beam was impingent on a $200 \mu \mathrm{g} / \mathrm{cm}^{2}{ }^{\text {nat }} \mathrm{Si}\left(\sim 92 \%{ }^{28} \mathrm{Si}\right)$ target, which was surrounded by two arrays of segmented silicon detectors, as shown in Figure 1. The Silicon Detector Array (SIDAR) [9] covering $\sim 18$ to $50^{\circ}\left(\sim 19-54^{\circ}\right.$ in the center of mass), was used to measure tritons from the (p,t) reaction at sixteen angles simultaneously. Protons from the decay of unbound states in ${ }^{26} \mathrm{Si}$ were measured between $\sim 50^{\circ}$ and $\sim 90^{\circ}$ in the laboratory using six $65 \mu \mathrm{m}$ non-resistive strip detectors of the Oak Ridge Rutgers University Barrel Array (ORRUBA) [10], covering roughly $6 \%$ of $4 \pi$ steradians.

Figure 2 displays the states in ${ }^{26} \mathrm{Si}$ above the proton threshold populated in the present work. Energies for levels in ${ }^{26} \mathrm{Si}$ taken from Seweryniak et al. [11] were used to calibrate each strip of SIDAR. Data taken from recent ${ }^{26} \mathrm{Si}$ mass measurements $[8,12]$ were used to determine the $(\mathrm{p}, \mathrm{t})$ reaction Q-value and the proton threshold in ${ }^{26} \mathrm{Si}(5513.7 \pm 0.5 \mathrm{keV})$. A few contaminant peaks from reactions on ${ }^{12} \mathrm{C}$ and ${ }^{16} \mathrm{O}$ [3] were identified by their different kinematics. No evidence of 


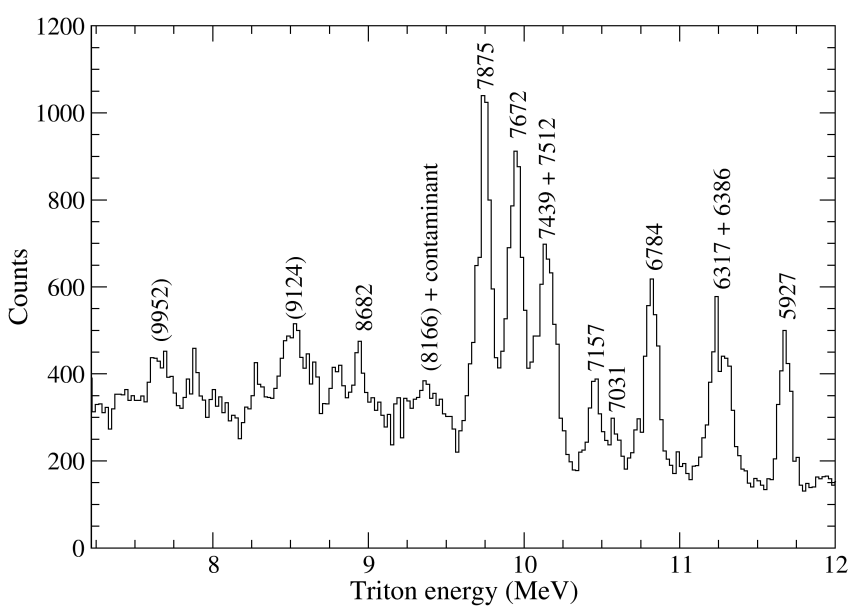

Figure 2: Triton spectrum for the SIDAR strip at $\theta_{l a b} \approx 23^{\circ}$, for populated levels above the proton threshold in ${ }^{26} \mathrm{Si}$.

(p,t) reactions on the contaminant ${ }^{29,30} \mathrm{Si}$ naturally occurring in the target was observed. Tritons populating higher-lying ${ }^{26} \mathrm{Si}$ excited states up to $\sim 10 \mathrm{MeV}$ were observed, some for the first time; a more detailed analysis may be found in Ref. [13]. There is good agreement between this work and the previous results $[1,3,11]$.

In addition to detecting tritons from the reaction in the forward-angle SIDAR detectors, decay protons from the excited ${ }^{26} \mathrm{Si}$ levels were detected in coincidence by the ORRUBA detectors. These events are visible in a plot of ORRUBA energy versus SIDAR energy shown in Figure 3. Using methodology similar to that of Deibel et al. [14], the present work is the first measurement proton branching ratios of proton-unbound states in ${ }^{26} \mathrm{Si}$, determined by examining the triton spectra as gated on these proton coincident events and correcting for efficiency.

Random coincidences between the SIDAR and ORRUBA detectors were reduced by requiring a proper energy correlation by implementing a 2D gate around the correlated events, demonstrated by the red box in Fig. 3. The number of additional random background events that fall within this gate were estimated by constructing equally-sized gates in various locations on events in the protonbound region of Fig. 3. The probability of a random coincidence was found to be $\sim 1 \%$ at most, and this random background was subsequently subtracted from later analysis. Accounting for this background, the extracted branching ratios were insensitive to small variations in the construction of the gate.

Angular correlation functions [15] for the decay protons were calculated [16] using scattering amplitudes from FRESCO [17] and the experimentally determined spin-parity and $\ell$-transfer information for the measured proton-unbound states, in order to determine the ORRUBA detection efficiency and thus the proton-branching ratios. The $2^{+}$and $3^{+}{ }^{26} \mathrm{Si}$ states which decay isotropically via $\ell=0$ transfers to the $5 / 2^{+}$ground state of ${ }^{25} \mathrm{Al}$ displayed direction-independent angular correlations, but branching ratios for states with anisotropic decay had to be adjusted for the angle-dependent detection efficiency; additionally, the ERESCO calculations took into account the necessary two-step processes to populate unnatural parity states [13]. The dominant uncertainty 


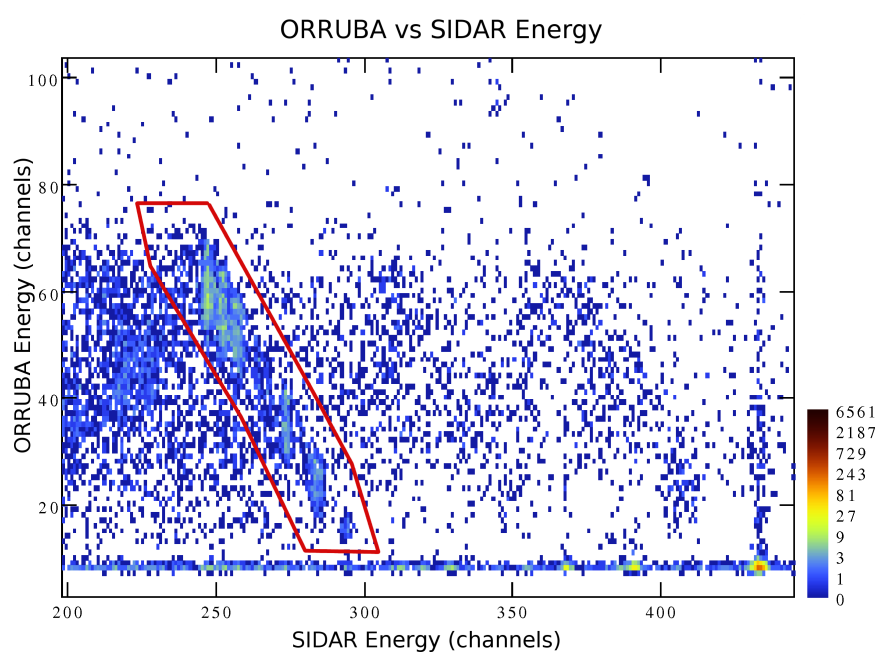

Figure 3: ORRUBA energy (vertical) vs. SIDAR energy (horizontal) for detected proton-triton coincident events. The red box gates the events associated with proton decay from excited ${ }^{26} \mathrm{Si}$ levels.

in the branching ratios from this work arises from statistics, though systematic uncertainties could arise from incorrect $J^{\pi}$ assignments.

The dominant contribution to the ${ }^{25} \mathrm{Al}(\mathrm{p}, \gamma){ }^{26} \mathrm{Si}$ reaction rate at astrophysical energies will be reactions preceding via the first $\ell=0$ resonance in the ${ }^{25} \mathrm{Al}+\mathrm{p}$ system to fall within the Gamow window. The location of this level in energy has been under some debate, and a recent $\gamma$-ray spectroscopy measurement [11] suggested this level is shifted by $\sim 5 \mathrm{keV}$ from previous placements, due to updated transition energies. A recent reanalysis [18] thus assigned a new energy of $E_{x}=5920.7 \pm 2.6 \mathrm{keV}\left(E_{r}=412 \pm 2 \mathrm{keV}\right)$ to the first $\ell=0$ resonance. The energy measured in the current work, which includes the updated ${ }^{26} \mathrm{Si}$ mass [8], is $E_{x}=5927 \pm 4 \mathrm{keV}\left(E_{r}=413 \pm 4 \mathrm{keV}\right)$, which is consistent with the reanalysis [13]. Furthermore, the current work determined the protondecay branching ratio for this state to be $0.91 \pm 0.10$ [13], in good agreement with the predicted branching ratio $[3,7]$ for a $3^{+}$level, supporting the spin and parity assignment.

A concurrent ${ }^{28} \mathrm{Si}(\mathrm{p}, \mathrm{t}){ }^{26} \mathrm{Si}$ measurement using the spectrometer at the Research Center for Nuclear Physics (RCNP) [19] also sought to confirm level energies and spin/parity assignments for states of astrophysical interest in ${ }^{26} \mathrm{Si}$. This work was unable to unambiguously see the mostimportant lowest $\ell=0$ level due to a strong contaminant line. However, the excitation energies measured for ${ }^{26} \mathrm{Si}$ levels in Ref. [19] are generally in agreement with the present results.

\section{Conclusion}

Resonances which affect the ${ }^{25} \mathrm{Al}(\mathrm{p}, \gamma)^{26} \mathrm{Si}$ reaction rate in astrophysical scenarios have been studied via the ${ }^{28} \mathrm{Si}(\mathrm{p}, \mathrm{t})^{26} \mathrm{Si} *(\mathrm{p})$ reaction. Proton branching ratios have been measured for the first time [13] for unbound ${ }^{26} \mathrm{Si}$ levels, by detecting the decay protons from several states in ${ }^{26} \mathrm{Si}$ in coincidence with tritons from the $(\mathrm{p}, \mathrm{t})$ reaction. The results of this work support the astrophysical conclusions in a number of recently published measurements and analyses [3, 7, 8, 18]. Further 
study of the proton and $\gamma$ partial widths of low-lying levels above the ${ }^{26} \mathrm{Si}$ proton threshold is required in order to reduce the remaining uncertainties in the astrophysical ${ }^{25} \mathrm{Al}(\mathrm{p}, \gamma){ }^{26} \mathrm{Si}$ reaction rate.

\section{Acknowledgments}

The authors wish to thank Dr. Carl Brune for his assistance with the decay angular correlation calculations. This research was supported in part by the National Nuclear Security Administration under the Stewardship Science Academic Alliances program through the U.S. DOE Cooperative Agreement DE-FG52-08NA28552 with Rutgers University and Oak Ridge Associated Universities. This work was also supported in part by the U.S. DOE under contract DE-FG02-96ER40955 with Tennessee Technological University, DE-FG02-96ER40983 with the University of Tennessee Knoxville, and by the National Science Foundation. Research sponsored by the Office of Nuclear Physics, U.S. Department of Energy.

\section{References}

[1] D. Bardayan et al., Phys. Rev. C65, 032801(R) (2002).

[2] J.C. Thomas et al., Eur. Phys. J. A 21, 419 (2004).

[3] D. Bardayan et al., Phys. Rev. C74, 045804 (2006).

[4] J.A. Caggiano et al., Phys. Rev. C65, 055801 (2002).

[5] Y. Parpottas et al., Phys. Rev. C70, 065805 (2004).

[6] Y. Parpottas et al., Phys. Rev. C73, 049907 (2006).

[7] P.N. Peplowski et al., Phys. Rev. C73, 049907 (2006).

[8] T. Eronen et al., Phys. Rev. C79, 032802(R) (2009).

[9] D. Bardayan et al., Phys. Rev. Lett. 83, pp45-48 (1999).

[10] S.D. Pain et al., Nucl. Instr. and Methods B261, pp1122 (2007).

[11] D. Seweryniak et al., Phys. Rev. C75, 062801 (2007).

[12] A.A. Kwiatkowski et al., Phys. Rev. C81, 058501 (2010).

[13] K.A. Chipps et al., Phys. Rev. C82, 045803 (2010).

[14] C.M. Deibel, J.A. Clark, R. Lewis, A. Parikh, P.D. Parker and C. Wrede, Phys. Rev. C80, 035806 (2009).

[15] G.R. Satchler, Direct Nuclear Reactions, Clarendon Press (c)1983.

[16] C.R. Brune, Private communication.

[17] I.J. Thompson, Comp. Phys. Rep.7, pp167-211 (1988).

[18] C. Wrede, Phys. Rev. C79, 035803 (2009).

[19] C. Matic et al., Phys. Rev. C82, 025807 (2010). 